\title{
Spinal muscular atrophy - new therapies, new challenges
}

\author{
Maria Jędrzejowska ${ }^{1}$, Anna Kostera-Pruszczyk ${ }^{2}$ \\ ${ }^{1}$ Neuromuscular Unit, Mossakowski Medical Research Centre, Polish Academy of Sciences, Warsaw, Poland \\ ${ }^{2}$ Department of Neurology, Medical University of Warsaw, Warsaw, Poland
}

\begin{abstract}
Spinal muscular atrophy (SMA) is a progressive neurodegenerative disease with an autosomal recessive trait of inheritance and great variability of its clinical course - from the lethal congenital type (SMA0) to the adult-onset form (SMA4). The disease is associated with a deficiency of SMN protein, which is encoded by two genes SMN1 and SMN2.

Clinical symptoms depend on mutations in the SMN1 gene. The number of copies of twin similar SMN2 gene, which produces small amounts of SMN protein, is the main phenotype modifier, which determines the clinical severity of the disease. Until recently, it was considered that spinal cord motoneurons undergo selective loss. Recent studies have shown the role of SMN protein in various cellular processes and the multisystemic character of SMA. The aim of the therapeutic strategies developed so far has been to increase the expression of SMN protein by modifying the splicing of SMN2 gene (intrathecally administered antisense oligonucleotide - nusinersen; orally available small molecules: RG7916 and LMI070 or SMN1 gene replacement therapy (AAV9-SMN).

The first SMN2-directed antisense oligonucleotide (nusinersen) has demonstrated in clinical trials high efficiency, and it has now been registered. The best effects were obtained in patients who were introduced to the drug in the pre-symptomatic period. Studies on other substances are ongoing. The great advances in SMA therapy and increased understanding of the pathogenesis of the disease raise hopes for changes to the natural history of the disease. Simultaneously, it increases awareness of the need to improve the standard of patient care and early diagnosis (newborn screening). Many questions (e.g. emerging phenotypes, combined therapies, systemic vs. intrathecal administration, long-term consequences, and complications of the therapy) will require further studies and observations.
\end{abstract}

Key words: spinal muscular atrophy, SMN1 gene, SMN protein, nusinersen, gene therapy

(Neurol Neurochir Pol 2020; 54 (1): 8-13)

\section{Introduction}

Spinal muscular atrophy belongs to a group of diseases characterised by a loss of spinal cord motoneurons, which leads to skeletal muscle atrophy and weakness.

The most common form is proximal spinal muscular atrophy infantile and juvenile type, associated with mutations in the SMN1 gene. In most cases, SMA is a severe, progressive and incurable disease, which leads to paralysis and respiratory failure. Despite improved standards of care, it remains one of the most frequent causes of death in children affected with genetic disorders.
Since the identification of the SMN1 gene, associated with pathogenesis of the diseases, intensive work has been undertaken with the aim of expanding knowledge of the molecular defect and pathogenesis of the disease, its natural history, and potential therapeutic methods [1]. Recently, studies on the first drug in SMA - the antisense oligonucleotide nusinersen - have been successfully completed $[2,3]$. Research into, it is to be hoped, equally effective substances with various mechanisms of action is underway (gene therapy - AVXS-101, micromolecules targeting alternative splicing of SMN2: RO7034067, LMI070) [4]. Encouraging results from preliminary tests have raised hopes for their effectiveness, and thereby changing the

Address for correspondence: Anna Kostera-Pruszczyk, Department of Neurology, Medical University of Warsaw, Banacha 1a Str., 02-097 Warsaw, Poland, e-mail: anna.kostera-pruszczyk@wum.edu.pl 
natural history of this severe and incurable disease, albeit many questions remain to be answered.

\section{Classification of SMA}

The currently approved division of SMA expands the classic classification by Munsat (into SMA1, 2 and 3 ) by the addition of two further forms: SMA0 with prenatal onset of clinical symptoms; and the adult form SMA4 [5].

SMA0 is a rare form, with symptoms visible already during foetal life and a poor prognosis [6]. Weak foetal movements are noted by mothers-to-be in the prenatal period. After birth, hypotonia, areflexia, a total or almost total lack of active movement, and very often arthrogryposis are observed. Respiratory failure develops in the first minutes or hours of life. The disease is associated with an extreme deficit of the SMN protein.

SMA1 patients present within the first six months of life with progressive generalised muscle weakness, atrophy and areflexia, and patients are never able to sit unsupported. SMA1 leads to respiratory insufficiency within the first year of life.

In SMA2, the first symptoms are seen between the ages of 7-18 months; the child is never able to stand but can sit unsupported. Later in life, severe scoliosis and contractures develop.

SMA3 is a chronic form, symptomatic after 18 months of life. Independent walking is achieved, but many patients lose ambulation $[5,6]$.

At the other end of the disease severity spectrum there is its adult form, SMA4, where onset usually occurs in the third decade of life [7]. This is characterised by slowly progressive weakness of proximal muscles, mainly quadriceps, with a good prognosis for life and preserved respiratory function. A novelty in the current classification is the division of the first and third forms into subtypes $a, b$, and $c$, depending on age at onset of the first symptoms [8]. It has been noted that earlier onset of the disease usually determines a more severe course. It seems that this classification is a compromise between a too general division into basic forms and a too detailed and difficult in everyday practice classification according to Dubowitz $(1.1-1.9,2.1-2.9,3.1-3.9,4)$ [9]. At the same time, it should be remembered that SMA phenotypes create a clinical continuum from the congenital to adult forms, and even asymptomatic cases. Thus within each form, cases with a milder or a more severe course of the disease will be seen. But there are also borderline cases which do not allow easy and unequivocal qualification to any basic group.

\section{Epidemiology}

Recently published study results on SMA epidemiology in Europe indicate an SMA incidence of 1:8,400 births (11.9/100,000) [10]. In 2011-2015 SMA was diagnosed in 4,653 patients in Europe, with 992 cases in 2015 alone. These results are compatible with the largest study on SMA epidemiology ever initiated, which has been conducted in the USA on a multi-ethnic group of 68,478 people [11]. This showed an SMA carrier frequency in the population of 1:54 people and an incidence of 1:11,000 births. A significant difference in carrier frequency was noted as depending on race, with the highest in Caucasians (2.02\%) and the lowest in African-Americans (0.98\%).

In contrast to SMA incidence, its prevalence is difficult to estimate. This is due to the various ages of onset and clinical courses of SMA which influence lifespan, additionally modified by improved standards of care, especially respiratory support. The global register of patients (the TREAT-NMD Global SMA Patient Registry) provided data from 26 national registries, representing 29 countries and contained a total of 4,526 genetically confirmed patients. This number does not reflect the real number of patients suffering from SMA worldwide $[10,12]$.

\section{The natural history of SMA}

Spinal muscular atrophy is characterised by a very wide spectrum of disease onset, symptom severity, and type of complications. Thus the natural history of the disease might vary in a given patient. Its clinical course depends on the quality of medical care, prophylaxis, and, above all, physiotherapy and appropriate treatment of complications. Recent studies have shown some relationships. In the SMA1 form, the earlier the onset of symptoms, the worse the prognosis and the shorter the survival. A study on the trajectories of SMA1 progression revealed baseline CHOP INTEND scores of below 20 in SMA1a and of 20-40 for both SMA1b and SMA1c [13]. The rate of progression in SMAla, b and c was significantly different, but none of the children showed any improvement. All infants with the most severe phenotype had rapid declines, and none survived beyond 13 months. Among children with type 1 of SMA, the number of SMN2 copies is a strong biomarker and disease modifier - the survival curve is decreased compared to patients with two copies. Median survival or age of introduction of mechanical ventilation ( $>16$ hours a day) is 13.5 months [14]. This period decreases to 10.5 months in children with two copies of SMN2. However, it should be noted that the presented data was obtained in a centre which uses the proactive therapeutic approach (i.e. pre-symptomatic mechanical clearing of respiratory tract with cough assistor, NIV, tube feeding or through PEG). Older reports, or reports from centres which focus on palliative treatment, indicate median survival of 6-7 months for SMA1 [15]. According to Australian studies, the chances of survival at $1,2,4$, and 10 years for children with SMA1 are 40\%, 25\%, 6\%, and 0\%, respectively [16]. In turn, a Polish-German study from 2002 indicated that only $10 \%$ of SMA1 patients reach the age of 5 years [17]. Respiratory and feeding support increases survival by months and indeed years, but at the price of full dependence on gastrostomy and NIV/IV, with no improvement of motor function [18]. 
The prognosis for life in patients with SMA2 is much better. According to Australian studies, almost 93\% of these patients reach the age of 20 years and approximately $52 \%$ survive until the age of at least 40. The Polish-German study from 1997 on a group of 240 SMA2 patients suggested a survival probability of about $75 \%$ at the age of 20 years [19]. The survival curve for patients with SMA3 and SMA4 seems to be the same as the average for the general population. In contrast to the acute form of SMA, patients with chronic forms can acquire new motor skills. In patients unable to walk, functional improvement is usually seen until the age of 4-5 years, while between the ages of 5 and 15 years they often experience a gradual deterioration but eventual stabilisation of motor function [20]. Adolescence seems to be the most difficult period for some SMA patients, as many of them lose the ability to walk independently. The probability of preserving the ability to independently walk after 10, 20 and 40 years of the disease is $73 \%, 44 \%$, and $34 \%$, respectively, for group 3a and $97 \%, 89 \%$, and $67 \%$, respectively, for group 3b [19].

\section{Standards of care}

Spinal muscular atrophy is a progressive and multisystemic disease that leads to many complications: respiratory (impaired cough reflex, respiratory distress), gastrointestinal (gastro-oesophageal reflux, swallowing problems, malnutrition or obesity), musculoskeletal (scoliosis, joint contractures, hip dislocation), and others. Quality of care and prophylaxis of complications definitely prolong lifespan and improve the quality of everyday functioning. Therefore the introduction of uniform criteria of care in SMA worldwide would be of the highest importance. The first standards of care were developed by international experts in SMA and published in 2007 [21]. In 2017, the guidelines of therapy were updated. Recommendations include some aspects (classification and natural history, diagnostics and genetics, management of newly-diagnosed patients, pulmonary and acute care, medications, immunisations, orthopaedic management and rehabilitation, gastroenterological care, symptoms from other organs, ethics and palliative care). Recently published standards present precise recommendations regarding rehabilitation, pulmonary and orthopaedic management, depending on the functional status of the patient (i.e. non-sitters, sitters, and walkers) [22, 23].

Comprehensive care for SMA patients requires the involvement of a multidisciplinary team of specialists from various fields, including neurologists, orthopaedic surgeons, pulmonologists, gastroenterologists, anaesthesiologists, geneticists, physiotherapists and dieticians.

\section{Molecular pathogenesis}

The pathogenesis of spinal muscular atrophy is associated with two similar twin genes: SMN1 and SMN2. The clinical symptoms of SMA depend on mutations of the SMN1 gene, among which $96.5 \%$ is biallelic deletion of this gene [24]. Three percent of SMA patients bear point mutations, which occur in a compound heterozygous state with deletion. To date, more than 70 point mutations of the SMN1 gene have been described. In the Polish population, seven such variants have been identified. The most frequent is mutation T274I in exon 6, which is correlated with chronic forms of SMA [25].

The SMN2 gene acts as a phenotype modifier. Mutations of the SMN2 gene are not pathogenic and, as a homozygous deletion, have been found in approximately $10 \%$ of a healthy population [26]. However an increased number of SMN2 copies, as a result of duplication or conversion, alleviates the clinical course of SMA.

SMN1 and SMN2 genes are twin similar [27]. Their sequence functionally differs by only one substitution $\mathrm{C}$ in $\mathrm{T}$ in exon 7. In healthy people, the expression of both these genes SMN1 and SMN2 leads to the production of the protein SMN. In patients with spinal muscular atrophy, the SMN protein is expressed only from the preserved SMN2 gene. The SMN2 gene, due to a single-nucleotide difference in exon 7 , undergoes alternative splicing during posttranscriptional processing. Substitution C to T in codon 280 changes the sequence of exonic splice enhancer (ESE), cancelling its action. This in turn leads to the deletion of exon 7 and eventually the practical loss of functional SMN protein. A small amount of SMN2 transcript (approximately 10-20\%) undergoes normal splicing and produces full-length SMN protein, structurally and functionally identical to the product of SMN1 copy.

An increased number of SMN2 copies (through duplication or conversion of SMN1 into SMN2) can compensate for deficits caused by loss of the SMN1 gene, through an increased level of the full-length SMN protein in tissues. A strong correlation has been demonstrated between the number of SMN2 copies and the phenotype of the disease. In patients with an acute form of SMA 1-2, SMN2 copies are usually found, while in patients with an intermediate form 2-3 copies are usually found. With a mild form 3-4, and even 5-6 copies, are found [28-30].

The number of SMN2 copies does not fully explain the phenotype variability in SMA. A recently published study on a large number of patients showed that in a group of $1,627 \mathrm{pa}-$ tients and three SMN2 copies, SMA1, 2 and 3 were diagnosed in $15 \%, 54 \%$, and $31 \%$ respectively. This is probably due to the fact that SMN2 copies are not functionally equivalent and produce various amount of full-value SMN protein. This could be associated with the role of epigenetic factors, especially with a different pattern of SMN2 methylation. Significantly lower methylation has been found in patients with the mild form of SMA, compared to patients with the severe form who had the same number of SMN2 copies. However, considering the different level of methylation in various tissues, drawing final conclusions can be challenging.

Another phenotype modifier is substitution c.859G $>\mathrm{C}$ in exon 7 of SMN2 gene [31]. This has been described in several 
patients in whom a mild phenotype did not correlate with the number of SMN2 copies (one copy of SMN2 in patients with SMA2 or SMA3). This change creates a sequence of a new ESE in exon 7, therefore increasing the level of the full-length SMN protein and alleviating the symptoms of the disease.

However, this modifier is very rarely identified. In a cohort of 625 Spanish patients it was found in 10 cases with discordant phenotypes (four cases with SMA type 2 and two SMN2 copies, four cases with SMA3 and two SMN2 copies, and two cases with SMA3 and three SMN2 copies) [32]. Among the factors which influence SMA clinical course, sex has also been mentioned. It has been observed that girls are affected by the benign form half as frequently as boys, while cases with asymptomatic biallelic mutation of SMN1 are more frequent in women [33]. In asymptomatic female carriers of SMN1 deletion, whose brothers had SMA, overexpression of plastin 3, encoded by PLS3 gene on chromosome X, has been found [34]. Another newly discovered SMA modifier is NCALD, a $\mathrm{Ca}^{2+}$-dependent negative regulator of endocytosis. Unlike PLS3, which alleviates SMA upon overexpression, NCALD reduction protects individuals with four SMN2 copies from developing SMA. NCALD transcript showed a 4 - to 5-fold downregulation in the asymptomatic members of a USA family versus SMA1 cases from this family or an independent type 3 SMA group [35].

While the genetic basis of SMA is well understood, the specific molecular pathways underlying SMA are still not completely understood. SMN protein modulates almost every aspect of RNA metabolism, including transcription, splicing, biogenesis of snRNPs, snoRNP assembly, telomerase activity, translation, and mRNA transport. Biogenesis of the spliceosomal snRNP assembly is the best characterised function of SMN. The SMN protein, together with some other proteins such as Gemins 2-8 and Unrip creates a multiprotein complex which is found both in cytoplasm and in nucleus. This complex is involved in the assembly of snRNP, an essential component of pre-mRNA splicing machinery in cells [36].

Independently of the SMN involvement in RNA metabolism, SMN regulates also DNA repair, cell signalling, endocytosis, autophagy and other functions. It is not known which function is crucial for SMA symptoms. It is likely that SMN deficiency simultaneously impacts upon multiple functions.

Since the discovery of the basic function of the SMN protein, there has been discussion as to why deficiency of the protein, playing such an important role in the metabolism, causes a near selective loss of motoneurons. SMN protein level in tissues might be critical. Animal models have shown that total loss of SMN protein is lethal in the foetal period. In knock-out mice without their own $S M N$ gene, and with introduced human SMN2, the severity of the disease depends on the number of introduced copies of the SMN2 gene. Mice with two copies are affected very severely, yet those with eight copies do not show any symptoms of SMA. A similar result has been obtained by increasing SMN2 expression through a prion promoter selectively within the central nervous system in mice with two copies of SMN2. These mice are healthy. Therefore it seems that the majority of tissues require for normal function only a low level of SMN, while the CNS needs much more.

While SMA mouse models illustrate the importance of SMN in the nervous system, recent studies have indicated a significant effect of SMN protein deficiency on the development and function of other organs i.e. bone, lungs, pancreas, cardiovascular system, liver, and spleen. Observations of symptoms occurring in patients with SMA seem to confirm these results. Thus, as SMA begins to be treated as a multi-systemic disease, it may well require systemic treatment.

\section{New therapies}

Recent years have brought dynamic progress in the development of novel SMA therapies. Nusinersen (Spinraza) is a survival motor neuron-2 (SMN2)-directed antisense oligonucleotide. It binds to the SMN2 pre-mRNA and promotes inclusion of exon 7, resulting in increased SMN protein production. The drug does not cross the blood-brain barrier and is delivered by intrathecal injection.

The safety and efficacy of nusinersen were demonstrated in phase I-III studies leading to early termination of a clinical trial. Nusinersen was licensed for the treatment of all types of SMA in December 2016 in the USA, and a few months later in the EU. The drug has become available to a wide SMA population. Recent reports demonstrate ongoing benefits for patients treated for over three years, as well as for patients who started treatment in the more advanced stages of the disease $[37,38]$. Several promising molecules are in phase II or III clinical trials. Risdiplam and branaplam are orally administered small molecules that act as SMN2 gene splicing modifiers [39-41].

Gene therapy is another emerging therapeutic option. Nonpathogenic, nonreplicating AAV parvovirus has been engineered to become a vector delivering full-length SMN1 cDNA to the host cells [8]. Preclinical studies have demonstrated the safety and efficacy of AAV9 in animal models of SMA [42]. Single-dose gene replacement resulted in the development of motor milestones in SMA1 children treated with a high dose, as reflected in the CHOP-INTEND scores. Two of the children started walking, and 11/12 were able to sit unassisted. All were alive at 20 months of age, compared to $8 \%$ in the historical controls [43]. The pulmonary and nutritional status of the children was also significantly improved [44]. Functional improvement was more pronounced in infants treated before they were three months old [45]. In the light of these results, the USA Food and Drug Administration recently approved onasemnogene abeparvovec-xioi, an adeno-associated virus vector-based as the first ever gene therapy for children with SMA below the age of two years.

All currently conducted clinical trials can be followed on the clinicaltrials.gov website. 


\section{Conclusions}

SMA is a unique disease, since SMN2 copy of the gene is present in all patients, being the most promising therapeutic target. The first antisense-based drug targeting intronic splicing silencer N1 in SMN2 gene, nusinersen, was discovered, tested and registered within a short time. The next emerging therapies are under advanced research and clinical trials. The efficacy of the developed treatment methods is the cause of considerable excitement. But many questions still remain concerning long-term effectiveness, emerging phenotypes, safety profile and tolerance, drug effectiveness in chronic forms, concomitant administration of medicines with various mechanisms of action, administration routes, and the correct time of treatment in the mild forms. Treatment costs can be also a challenge for healthcare systems. Animal studies suggesting the multisystemic character of the disease might indicate that intrathecal administration of the SMA therapies may not be fully effective. The high demand for SMN protein in early life indicates the crucial role of an early diagnosis of this disease for maximum effectiveness. This could be achieved by neonatal screening, although for the severe form this could still be too late. Population screening towards a carrier state of SMA should be considered. At present, from the clinical point of view, the most important seems to be detailed observations of the patients treated with the innovative therapies, and strict compliance with the SMA standards of care.

Funding: This publication was prepared without any external source of funding.

Conflict of interest: Anna Kostera-Pruszczyk: Advisory Board, lecture honoraria, grant and travel support: Biogen, Advisory Board and lecture suport: PTC Therapeutics, travel suport: Roche.Maria Jędrzejowska: Advisory Board, lecture honoraria, travel support: Biogen

\section{References}

1. Lefebvre S, Bürglen L, Reboullet S, et al. Identification and characterization of a spinal muscular atrophy-determining gene. Cell. 1995; 80(1): 155-165, doi: 10.1016/0092-8674(95)90460-3, indexed in Pubmed: 7813012.

2. Mercuri E, Darras BT, Chiriboga CA, et al. CHERISH Study Group. Nusinersen versus Sham Control in Later-Onset Spinal Muscular Atrophy. N Engl J Med. 2018; 378(7): 625-635, doi: 10.1056/NEJMoa1710504, indexed in Pubmed: 29443664.

3. Finkel RS, Mercuri E, Darras BT, et al. ENDEAR Study Group. Nusinersen versus Sham Control in Infantile-Onset Spinal Muscular Atrophy. N Engl J Med. 2017; 377(18): 1723-1732, doi: 10.1056/ NEJMoa1702752, indexed in Pubmed: 29091570.

4. Kariyawasam D, Carey KA, Jones KJ, et al. New and developing therapies in spinal muscular atrophy. Paediatr Respir Rev. 2018; 28: 3-10, doi: 10.1016/j.prrv.2018.03.003, indexed in Pubmed: 29703692.
5. Munsat TL, Davies KE. International SMA consortium meeting. (2628 June 1992, Bonn, Germany). Neuromuscul Disord. 1992; 2(56): 423-428, doi: 10.1016/s0960-8966(06)80015-5, indexed in Pubmed: 1300191.

6. MacLeod MJ, Taylor JE, Lunt PW, et al. Prenatal onset spinal muscular atrophy. Eur J Paediatr Neurol. 1999; 3(2): 65-72, doi: 10.1053/ ejpn.1999.0184, indexed in Pubmed: 10700541.

7. Brahe C, Servidei S, Zappata S, et al. Genetic homogeneity between childhood-onset and adult-onset autosomal recessive spinal muscular atrophy. Lancet. 1995; 346(8977): 741-742, doi: 10.1016/s01406736(95)91507-9, indexed in Pubmed: 7658877.

8. Farrar MA, Park SB, Vucic S, et al. Emerging therapies and challenges in spinal muscular atrophy. Ann Neurol. 2017; 81(3): 355-368, doi: 10.1002/ana.24864, indexed in Pubmed: 28026041.

9. Dubowitz V. Chaos in the classification of SMA: a possible resolution. Neuromuscul Disord. 1995; 5(1): 3-5, doi: 10.1016/09608966(94)00075-k, indexed in Pubmed: 7719138.

10. Verhaart IEC, Robertson A, Leary R, et al. A multi-source approach to determine SMA incidence and research ready population. J Neurol. 2017; 264(7): 1465-1473, doi: 10.1007/s00415-017-8549-1, indexed in Pubmed: 28634652.

11. Sugarman $\mathrm{EA}$, Nagan $\mathrm{N}$, Zhu $\mathrm{H}$, et al. Pan-ethnic carrier screening and prenatal diagnosis for spinal muscular atrophy: clinical laboratory analysis of $>72,400$ specimens. Eur J Hum Genet. 2012; 20(1): 27-32, doi: 10.1038/ejhg.2011.134, indexed in Pubmed: 21811307.

12. Bladen $\mathrm{CL}$, Thompson R, Jackson JM, et al. Mapping the differences in care for 5,000 spinal muscular atrophy patients, a survey of 24 national registries in North America, Australasia and Europe. J Neurol. 2014; 261(1): 152-163, doi: 10.1007/s00415-013-7154-1, indexed in Pubmed: 24162038.

13. De Sanctis R, Pane M, Coratti G, et al. Clinical phenotypes and trajectories of disease progression in type 1 spinal muscular atrophy. Neuromuscul Disord. 2018; 28(1): 24-28, doi: 10.1016/j. nmd.2017.09.015, indexed in Pubmed: 29174525.

14. Finkel RS, McDermott MP, Kaufmann P, et al. Observational study of spinal muscular atrophy type I and implications for clinical trials. Neurology. 2014; 83(9): 810-817, doi: 10.1212/ WNL.0000000000000741, indexed in Pubmed: 25080519.

15. Cobben JM, Lemmink HH, Snoeck I, et al. Survival in SMA type I: a prospective analysis of 34 consecutive cases. Neuromuscul Disord. 2008; 18(7): 541-544, doi: 10.1016/j.nmd.2008.05.008, indexed in Pubmed: 18579378.

16. Farrar MA, Vucic S, Johnston HM, et al. Pathophysiological insights derived by natural history and motor function of spinal muscular atrophy. J Pediatr. 2013; 162(1): 155-159, doi: 10.1016/j. jpeds.2012.05.067, indexed in Pubmed: 22809660.

17. Borkowska J, Rudnik-Schoneborn S, Hausmanowa-Petrusewicz I, et al. Early infantile form of spinal muscular atrophy (Werdnig-Hoffmann disease) with prolonged survival. Folia Neuropathol. 2002; 40(1): 19-26, indexed in Pubmed: 12121035.

18. Bach JR, Saltstein K, Sinquee D, et al. Long-term survival in WerdnigHoffmann disease. Am J Phys Med Rehabil. 2007; 86(5): 339-45 quiz 346, doi: 10.1097/PHM.0b013e31804a8505, indexed in Pubmed: 17449977.

19. Zerres K, Rudnik-Schöneborn S, Forrest E, et al. A collaborative study on the natural history of childhood and juvenile onset proximal spinal muscular atrophy (type II and III SMA): 569 patients. J Neurol Sci. 1997; 146(1): 67-72, doi: 10.1016/s0022-510x(96)00284-5, indexed in Pubmed: 9077498 
20. Mercuri E, Finkel R, Montes J, et al. Patterns of disease progression in type 2 and 3 SMA: Implications for clinical trials. Neuromuscul Disord. 2016; 26(2): 126-131, doi: 10.1016/j.nmd.2015.10.006, indexed in Pubmed: 26776503.

21. Wang $\mathrm{CH}$, Finkel RS, Bertini ES, et al. Participants of the International Conference on SMA Standard of Care. Consensus statement for standard of care in spinal muscular atrophy. J Child Neurol. 2007; 22(8): 1027-1049, doi: 10.1177/0883073807305788, indexed in Pubmed: 17761659.

22. Finkel RS, Mercuri E, Meyer OH, et al. SMA Care group. Diagnosis and management of spinal muscular atrophy: Part 2: Pulmonary and acute care; medications, supplements and immunizations; other organ systems; and ethics. Neuromuscul Disord. 2018; 28(3): 197-207, doi: 10.1016/j.nmd.2017.11.004, indexed in Pubmed: 29305137.

23. Mercuri E, Finkel RS, Muntoni F, et al. SMA Care Group. Diagnosis and management of spinal muscular atrophy: Part 1: Recommendations for diagnosis, rehabilitation, orthopedic and nutritional care. Neuromuscul Disord. 2018; 28(2): 103-115, doi: 10.1016/j. nmd.2017.11.005, indexed in Pubmed: 29290580.

24. Jedrzejowska M, Wiszniewski W, Zimowski J, et al. Application of a rapid non-invasive technique in the molecular diagnosis of spinal muscular atrophy (SMA). Neurol Neurochir Pol. 2005; 39(2): 89-94, indexed in Pubmed: 15871052.

25. Jędrzejowska M, Gos M, Zimowski JG, et al. Novel point mutations in survival motor neuron 1 gene expand the spectrum of phenotypes observed in spinal muscular atrophy patients. Neuromuscul Disord. 2014; 24(7): 617-623, doi: 10.1016/j.nmd.2014.04.003, indexed in Pubmed: 24844453.

26. Jedrzejowska M, Milewski M, Zimowski J, et al. Phenotype modifiers of spinal muscular atrophy: the number of SMN2 gene copies, deletion in the NAIP gene and probably gender influence the course of the disease. Acta Biochim Pol. 2009; 56(1): 103-108, indexed in Pubmed: 19287802.

27. Farrar MA, Johnston HM, Grattan-Smith P, et al. Spinal muscular atrophy: molecular mechanisms. Curr Mol Med. 2009; 9(7): 851-862, doi: 10.2174/156652409789105516, indexed in Pubmed: 19860664.

28. Feldkötter M, Schwarzer V, Wirth R, et al. Quantitative analyses of SMN1 and SMN2 based on real-time lightCycler PCR: fast and highly reliable carrier testing and prediction of severity of spinal muscular atrophy. Am J Hum Genet. 2002; 70(2): 358-368, doi: 10.1086/338627, indexed in Pubmed: 11791208.

29. Wirth B, Brichta L, Schrank B, et al. Mildly affected patients with spinal muscular atrophy are partially protected by an increased SMN2 copy number. Hum Genet. 2006; 119(4): 422-428, doi: 10.1007/s00439006-0156-7, indexed in Pubmed: 16508748.

30. Mailman MD, Heinz JW, Papp AC, et al. Molecular analysis of spinal muscular atrophy and modification of the phenotype by SMN2. Genet Med. 2002; 4(1): 20-26, doi: 10.1097/00125817-20020100000004, indexed in Pubmed: 11839954.

31. Bernal S, Alías L, Barceló MJ, et al. The c.859G >C variant in the SMN2 gene is associated with types II and III SMA and originates from a common ancestor. J Med Genet. 2010; 47(9): 640-642, doi: 10.1136/ jmg.2010.079004, indexed in Pubmed: 20577007.

32. Calucho M, Bernal S, Alías L, et al. Correlation between SMA type and SMN2 copy number revisited: An analysis of 625 unrelated Spanish patients and a compilation of 2834 reported cases. Neuromuscul Disord. 2018; 28(3): 208-215, doi: 10.1016/j.nmd.2018.01.003, indexed in Pubmed: 29433793.
33. Hausmanowa-Petrusewicz I, Zaremba J, Borkowska J, et al. Chronic proximal spinal muscular atrophy of childhood and adolescence: sex influence. J Med Genet. 1984; 21(6): 447-450, doi: 10.1136/ jmg.21.6.447, indexed in Pubmed: 6512833.

34. Oprea GE, Kröber S, McWhorter ML, et al. Plastin 3 is a protective modifier of autosomal recessive spinal muscular atrophy. Science. 2008; 320(5875): 524-527, doi: 10.1126/science.1155085, indexed in Pubmed: 18440926.

35. Riessland M, Kaczmarek A, Schneider S, et al. Neurocalcin Delta Suppression Protects against Spinal Muscular Atrophy in Humans and across Species by Restoring Impaired Endocytosis. Am J Hum Genet. 2017; 100(2): 297-315, doi: 10.1016/j.ajhg.2017.01.005, indexed in Pubmed: 28132687.

36. Hosseinibarkooie S, Schneider S, Wirth B. Advances in understanding the role of disease-associated proteins in spinal muscular atrophy. Expert Rev Proteomics. 2017; 14(7): 581-592, doi: 10.1080/14789450.2017.1345631, indexed in Pubmed: 28635376.

37. Darras BT, Chiriboga CA, lannaccone ST, et al. ISIS-396443-CS2/ ISIS-396443-CS12 Study Groups. Nusinersen in later-onset spinal muscular atrophy: Long-term results from the phase $1 / 2$ studies. Neurology. 2019; 92(21): e2492-e2506, doi: 10.1212/ WNL.0000000000007527, indexed in Pubmed: 31019106.

38. Aragon-Gawinska K, Seferian AM, Daron A, et al. Nusinersen in patients older than 7 months with spinal muscular atrophy type 1: A cohort study. Neurology. 2018; 91(14): e1312-e1318, doi: 10.1212/ WNL.0000000000006281, indexed in Pubmed: 30158155.

39. Poirier A, Weetall M, Heinig K, et al. Risdiplam distributes and increases SMN protein in both the central nervous system and peripheral organs. Pharmacol Res Perspect. 2018; 6(6): e00447, doi: 10.1002/ prp2.447, indexed in Pubmed: 30519476.

40. Ratni H, Ebeling M, Baird J, et al. Discovery of Risdiplam, a Selective Survival of Motor Neuron-2 ( SMN2) Gene Splicing Modifier for the Treatment of Spinal Muscular Atrophy (SMA). J Med Chem. 2018; 61(15): 6501-6517, doi: 10.1021/acs.jmedchem.8b00741, indexed in Pubmed: 30044619.

41. Cheung AK, Hurley B, Kerrigan R, et al. Discovery of Small Molecule Splicing Modulators of Survival Motor Neuron-2 (SMN2) for the Treatment of Spinal Muscular Atrophy (SMA). J Med Chem. 2018; 61(24): 11021-11036, doi: 10.1021/acs.jmedchem.8b01291, indexed in Pubmed: 30407821.

42. Meyer K, Ferraiuolo L, Schmelzer L, et al. Improving single injection CSF delivery of AAV9-mediated gene therapy for SMA: a dose-response study in mice and nonhuman primates. Mol Ther. 2015; 23(3): 477 487, doi: 10.1038/mt.2014.210, indexed in Pubmed: 25358252.

43. Mendell JR, Al-Zaidy S, Shell R, et al. Single-Dose Gene-Replacement Therapy for Spinal Muscular Atrophy. N Engl J Med. 2017; 377(18): 1713-1722, doi: 10.1056/NEJMoa1706198, indexed in Pubmed: 29091557.

44. Al-Zaidy S, Pickard AS, Kotha K, et al. Health outcomes in spinal muscular atrophy type 1 following AVXS-101 gene replacement therapy. Pediatr Pulmonol. 2019; 54(2): 179-185, doi: 10.1002/ppul.24203, indexed in Pubmed: 30548438.

45. Lowes LP, Alfano LN, Arnold WD, et al. Impact of Age and Motor Function in a Phase 1/2A Study of Infants With SMA Type 1 Receiving Single-Dose Gene Replacement Therapy. Pediatr Neurol. 2019; 98 : 39-45, doi: 10.1016/.jpediatrneurol.2019.05.005, indexed in Pubmed: 31277975. 